Documentation et bibliothèques

DOCUMENTATION BIBLIOTHEQUES

\title{
Évaluation de l'efficacité de la bibliothèque : analyse des études majeures
}

\section{André Cossette}

Volume 24, numéro 3, septembre 1978

URI : https://id.erudit.org/iderudit/1055163ar

DOI : https://doi.org/10.7202/1055163ar

Aller au sommaire du numéro

\section{Éditeur(s)}

Association pour l'avancement des sciences et des techniques de la documentation (ASTED)

\section{ISSN}

0315-2340 (imprimé)

2291-8949 (numérique)

Découvrir la revue

\section{Citer cet article}

Cossette, A. (1978). Évaluation de l'efficacité de la bibliothèque : analyse des études majeures. Documentation et bibliothèques, 24(3), 115-128.

https://doi.org/10.7202/1055163ar

\section{Résumé de l'article}

L'évaluation scientifique de l'efficacité d'une bibliothèque exige la mesure de ses performances documentaires au moyen d'indicateurs de résultats. Les études d'évaluation en bibliothéconomie reposent sur l'approche systémique (recherche opérationnelle) et se servent de modèles analytiques pour quantifier la capacité des centres documentaires de répondre adéquatement aux multiples besoins des usagers. L'auteur effectue une analyse critique des principales études de performance ayant pour but de mesurer l'efficacité d'une bibliothèque qui doit répondre aux requêtes documentaires exprimées, soit par l'auteur ou le titre, soit par un sujet. L'emploi de méthodes quantitatives dans les projets d'évaluation des bibliothèques permettra d'améliorer la qualité des services documentaires et de modifier l'image de la bibliothéconomie.
Tous droits réservés $@$ Association pour l'avancement des sciences et des techniques de la documentation (ASTED), 1978
Ce document est protégé par la loi sur le droit d'auteur. L'utilisation des services d'Érudit (y compris la reproduction) est assujettie à sa politique d'utilisation que vous pouvez consulter en ligne.

https://apropos.erudit.org/fr/usagers/politique-dutilisation/ 


\title{
Evaluation de l'efficacité de la bibliothèque: analyse des études majeures
}

\author{
André Cossette \\ Bibliothèque \\ Collège de Trois-Rivières
}

L'évaluation scientifique de l'efficacité d'une bibliothèque exige la mesure de ses performances documentaires au moyen d'indicateurs de résultats. Les études d'évaluation en bibliothéconomie reposent sur l'approche systémique (recherche opérationnelle) et se servent de modèles analytiques pour quantifier la capacité des centres documentaires de répondre adéquatement aux multiples besoins des usagers. L'auteur effectue une analyse critique des principales études de performance ayant pour but de mesurer l'efficacité d'une bibliothèque qui doit répondre aux requêtes documentaires exprimées, soit par l'auteur ou le titre, soit par un sujet. L'emploi de méthodes quantitatives dans les projets d'évaluation des bibliothèques permettra d'améliorer la qualité des services documentaires et de modifier l'image de la bibliothéconomie.

The scientific evaluation of the effectiveness of a library demands the measurement of its documentary performances by the means of result indicators. Studies of evaluation in library science are based upon the systematic approach (operational research) and use analytical models to quantify the capacity of documentary centers toanswer adequately the multiple needs of users The author makes a critical analysis of the principal performance studies which aim at measuring the effectiveness of a library which must answer requests for materials requested by author, title or by subject. The use of quantitative mothods in library evaluation projects will lead to the betterment of the quality of documentary services and to a modified image of librarianship.

La evaluación cientifica de la eficiencia de una biblioteca exige la medida de sus performances documentales por indicadores de resultados. Los estudiós de evaluacion en biblioteconomía se basan en la investigación por sistemas (investigación operacional) y utilizan modelos analíticos para cuantiar la capacidad de los centros de documentatión a responder de modo adecuado a las necesidades multiples de los usuarios. El autor hace un análisis crítico de los estudios principales de performance, cuyo objetivo es medir la eficiencia de una biblioteca que debe responder a las consultas documentales expresadas por autor o título, o por tema. La utilización de métodos cuantitativos en proyectos de evaluación de las bibliotecas permitirá mejorar la cualidad de los servicios de documentación y modificar la imagen de la biblioteconomía.

Un nombre considérable d'études portant sur l'évaluation de l'efficacité des bibliothèques et des centres de documentation (information retrieval systems) ont été publiées depuis une quinzaine d'années. Parmi ces écrits, plusieurs possèdent les mêmes caractéristiques que la majorité des articles publiés dans les revues professionnelles, en ce sens qu'ils consistent en des expériences personnelles, des opinions ou des anecdotes, plutôt qu'en des analyses rigoureuses et systématiques utilisant un cadre scientifique valable.

Notre objectif est d'analyser les études majeures à caractère scientifique réalisées dans le domaine de l'évaluation de l'efficacité des centres documentaires. Nous croyons que l'analyse des études rigoureuses déjà effec- 
tuées permettra de préciser les variables à contrôler dans les futurs projets d'évaluation de l'efficacité des bibliothèques du Québec.

La plupart des études d'évaluation en bibliothéconomie ont utilisé soit l'analyse de systèmes, soit la recherche opérationnelle, deux approches qui désignent, à toutes fins pratiques, une même réalité 1 . En effet, toutes deux sont quantitatives et appliquent une méthode scientifique à la résolution des problèmes concrets auxquels sont confrontés les centres documentaires dans leur tentative d'améliorer leur efficacité. Dans les deux cas, la procédure consiste à construire un modèle analytique de nature mathématique qui intègre les variables ou facteurs majeurs se rapportant au problème en cours d'étude et qui permet de calculer la performance du système ou du sous-système analysé en fonction de l'interaction entre les variables quantifiées. La manipulation des variables est souvent utilisée pour découvrir quelles valeurs, en termes mathématiques, if faut donner à chacune d'elles pour obtenir les meilleurs résultats, l'efficacité optimale.

En bibliothéconomie, l'approche systémique vise à mesurer objectivement les performances d'une bibliothèque particulière pour vérifier si les résultats, les produits et les services réalisés (output) correspondent aux objectifs documentaires fixés ( résultats attendus). Dans le cas d'une microévaluation, l'objet de l'étude est d'effectuer une analyse diagnostique des produits et des services documentaires offerts à la clientèle dans le but d'identifier leurs déficiences et d'améliorer l'efficacité de la bibliothèque.

Les études de performance ont principalement porté jusqu'ici sur l'analyse objective et systématique de deux aspects majeurs des résultats d'une bibliothèque considérée comme un système de communication ${ }^{2}$ d'information enregistrée: a) les produits et les services offerts aux usagers qui expriment des requêtes de documents identifiés, par l'auteur ou par le titre; b) les produits et les services

1. Abraham Bookstein and Don R. Swanson, "Introduction", Library Quarterly, vol. 42, no. 2 (January 1972), 2.

2. J.M. Orr, Libraries as Communication Systems, Westport, Conn., Greenwood Press, 1977, 228 p. (Contributions in Librarianship and Information Science, no. 17). fournis aux usagers qui expriment des requêtes documentaires par sujet. L'importance accordée à l'analyse de ces deux situations, où il s'agit d'évaluer si les produits documentaires fournis à l'usager satisfont ses attentes, nous semble justifiée, la première fonction d'une bibliothèque consistant à satisfaire les besoins en documentation de sa clientèle. Comme ces deux situations sont distinctes et nécessitent l'utilisation de mesures différentes, nous allons les étudier séparément. Examinons d'abord la problématique des requêtes de documents identifiés.

\section{Evaluation de l'efficacité dans la livraison des ciocuments ("niveau de satisfaction ")}

Un des objectifs majeurs des bibliothèques est de satisfaire le plus possible les requêtes de documents identifiés, par l'auteur ou par le titre, exprimées par la clientèle. D'ailleurs, les collections doivent être évaluées en fonction de la proportion des demandes qu'elles peuvent satisfaire ${ }^{3}$. Pour évaluer la capacité d'une bibliothèque de répondre convenablement aux requêtes qui mentionnent l'auteur ou le titre, il faut mesurer quantitativement la proportion des volumes demandés qu'elle a été capable de communiquer aux usagers.

La mesure utilisée pour évaluer la capacité d'une bibliothèque de fournir à sa clientèle les documents connus a été désignée par diverses expressions: "capacité de livraison de documents » (document delivery capability), "niveau de satisfaction" (satisfaction level), "disponibilité des documents" (document availability), "échec des usagers" (readers' failure), "frustration de l'usager » (user frustration), etc. Malgré la grande variété de termes utilisés pour nommer une telle mesure, l'indicateur de performance est le même dans tous les cas et révèle la proportion des requêtes de documents identifiés qu'une bibliothèque particulière peut satisfaire.

La "capacité de livraison de documents" ou le "niveau de satisfaction " d'une bibliothèque est relativement facile à mesurer en

3. F.W. Lancaster, The Measurement and Evaluation of Library Services, Washington, D.C., Information Resources Press, 1977, p. 202. 
termes quantitatifs, bien qu'il soit plus complexe d'identifier et surtout de mesurer les facteurs qui l'affectent. Nous analyserons les principaux modèles analytiques disponibles qui servent à mesurer le "niveau de satisfaction" relatif à une bibliothèque particulière et permettent d'évaluer sa capacité de communiquer aux usagers les documents connus demandés.

\section{"Capacité de livraison de documents" de Orr}

Orr ${ }^{4}$ et son équipe de l'Institute for the Advancement of Medical Communications ( IAMC ) de la Bibliothèque nationale de médecine des Etats-Unis semblent avoir été les premiers à utiliser l'approche systémique pour mesurer quantitativement la capacité d'une bibliothèque de satisfaire les requêtes documentaires de sa clientèle. Orr a désigné sous le nom de "capacité de livraison de documents " la mesure de performance qu'il a retenue pour évaluer la capacité de certaines bibliothèques médicales américaines de répondre aux demandes documentaires qui ieur sont communiquées.

Pour mesurer ces résultats, Orr a construit un test de livraison de documents (document delivery test) qui évalue l'efficacité d'une bibliothèque médicale à partir de deux critères importants pour l'usager: a) la capacité de satisfaire les requêtes documentaires exprimées; b) le temps nécessaire pour fournir chaque document demandé. Ce test comprend 300 références bibliographiques établies à partir des documents cités dans des écrits ( thèses, articles, etc.) rédigés par les usagers de chaque bibliothèque évaluée. Ces 300 références constituent un échantillon établi au hasard des citations apparaissant dans les plus récentes publications du personnel médical de l'institution que dessert la bibliothèque.

Le test a été administré dans 92 bibliothèques de médecine des Etats-Unis par des bibliothécaires professionnels qui simulaient le comportement des usagers. L'évaluateur véri-

4. R.H. Orr et al., "Development of methodologic tools for planning and managing library services. II: Measuring a library's capability for providing documents ", Bulletin of the Medical Library Association, vol. 56, no. 3 (July 1968), 241-267. fiait la présence des 300 références de son échantillon dans le catalogue de la bibliothèque évaluée dans le but de déterminer la proportion des documents déjà acquis. II mesurait aussi le temps de livraison, vérifiant la disponibilité de chaque document au moment $d \in$ la recherche. Cinq états de disponibilité correspondant à cinq vitesses possibles de livraison avaient été préalablement identifiés et codés sur une échelle exponentielle de temps de livraison. La première vitesse de livraison ( $10^{1}$ minutes) était attribuée à un document immédiatement disponible sur les rayons, tandis que la dernière vitesse ( $10^{5}$ minutes, i.e. une semaine) correspondait à un document qui n'avait pas été acquis.

Après avoir identifié l'état de disponibilité des 300 documents recherchés et l'avoir traduit en un chiffre de un à cinq représentant sa vitesse de livraison, l'évaluateur exprimait les résultats de sa recherche au moyen d'un index de capacité (capability index) comprenant une échelle de 0 à 100 points. Si un évaluateur attribuait le compte maximum de 100 points à une bibliothèque, cela signifiait que cette dernière possédait tous les documents figurant dans le test et que ceux-ci étaient tous disponibles sur les rayons au moment de l'enquête. Des 92 bibliothèques médicales évaluées, l'index de capacité a varié entre 49 et 94 points.

La méthodologie de Orr, qui évalue l'efficacité d'une bibliothèque en mesurant le temps qu'elle prend pour fournir des documents connus, est basée sur le principe qu'une bibliothèque a pour objectif fondamental de communiquer le plus rapidement possible les documents demandés par sa clientèle.

Le test de livraison de documents repose sur les deux présupposés suivants: a) les références bibliographiques contenues dans le test constituent un échantillon représentatif des demandes actuelles des usagers; b) les résultats de la recherche documentaire effectuée par des bibliothécaires professionnels ne travaillant pas à la bibliothèque évaluée équivalent approximativement aux résultats qu'auraient obtenus de simples usagers. Or, ces deux présupposés constituent de sérieuses 
déficiences méthodologiques. Buckland ${ }^{5}$ qualifie la méthodologie utilisée par Orr de fondamentalement défectueuse, car l'échantillonnage est essentiellement biaisé: les citations apparaissant dans les publications rédigées par les usagers sont déterminées, dans une large mesure, par les documents contenus dans la collection de la bibliothèque qu'ils fréquentent.

Lancaster $^{6}$ semble partager ce point de vue lorsqu'il affirme qu'une enquête effectuée à partir des requêtes documentaires actuelles des usagers est supérieure au DDT (document delivery test ), puisçu'elle se base sur les demandes réelles de la clientèle. De plus, le test de Orr ne constitue qu'un outil de macroévaluation. II ne permet pas une microévaluation de la qualité d'une collection, ni une identification de ses faiblesses, qui pourraient conduire à des recommandations susceptibles d'améliorer son rendement dans l'avenir.

\section{«Niveau de satisfaction» de Buckland}

L'étude entreprise par Buckland ${ }^{7}$ et son équipe à la Bibliothèque de I'Université de Lancaster, en Grande-Bretagne, constitue une microévaluation intéressante de la qualité du service. La mesure de performance utilisée par Buckland pour évaluer la capacité de cette bibliothèque universitaire de satisfaire aux demandes de documents identifiés est connue sous l'expression de "niveau de satisfaction» (satisfaction level). L'indicateur de performance obtenu comme résultat d'une telle mesure est établi à partir des techniques de l'analyse de systèmes et indique la proportion des requêtes documentaires satisfaites.

Pour mesurer le "niveau de satisfaction", Buckland emploie un modèle intégrant un certain nombre de facteurs qui affectent la capacité d'une bibliothèque de communiquer les documents requis par les usagers. Pour

5. M.K. Buckland, "The management of libraries and information centers", in Annual Review of Information Science and Technology, vol. 9. Washington, D.C., American Society for Information Science, 1974 , p. 347

6. F.W. Lancaster, The Measurement and Evaluation,... p. 237.

7. M.K. Buckland, "An operations research study of a variable loan and duplication policy at the University of Lancaster", Library Quarterly, vol. 42, no. 1 (January 1972), 97-106. chaque niveau de popularité, établi à partir d'une analyse des statistiques de prêt, les facteurs qui influencent la disponibilité des volumes sont: a) la durée de la période de prêt; b) le nombre de volumes à consulter sur place; c) le nombre d'exemplaires d'un titre; d) la possibilité de réserver un volume qui n'est pas disponible sur les rayons. La modification d'une de ces variables quantifiées du modèle permet de prédire quelle sera la performance de la bibliothèque telle que mesurée par le "niveau de satisfaction».

Buckland 8 a dégagé les relations fort complexes existant entre la fréquence des demandes d'un titre (sa popularité) et la période de temps pendant laquelle un volume n'est pas disponible sur les rayons.

a) Les chances d'un usager de trouver sur les rayons un exemplaire d'un titre désiré sont inversement proportionnelles à sa popularité, quelle que soit la durée de la période de prêt; plus un titre est en demande, plus le "niveau de satisfaction » est bas; moins un titre est en demande, plus le «niveau de satisfaction » est élevé.

b) La durée de la période de prêt et le « niveau de satisfaction » sont inversement proportionnels, quel que soit le niveau de popularité du document; plus la période de prêt est longue, plus le "niveau de satisfaction » est bas; plus la période de prêt est courte, plus le «niveau de satisfaction " est élevé.

c) La popularité et la durée de la période de prêt sont inversement proportionnelles, quel que soit le «niveau de satisfaction"; plus la popularité est grande, plus la période de prêt est courte; plus la popularité est faible, plus la période de prêt est longue.

d) Une augmentation du nombre d'exemplaires et une réduction de la durée de la période de prêt augmentent le "niveau de satisfaction».

Le "niveau de satisfaction» mesuré à la Bibliothèque de l'Université de Lancaster a été relativement bas, soit environ $60 \%$. Cela signifie que seulement six usagers sur dix obtiennent les volumes désirés. Les enquêtes effectuées depuis lors tendent à démontrer que d'aussi piètres résultats ne sont pas particuliers à cette bibliothèque universitaire, mais sont très fréquents et même inférieurs dans la

8. Ibid., 99. 
plupart des bibliothèques académiques. Un niveau de performance aussi médiocre a permis de découvrir qu'il existe présentement dans les bibliothèques une véritable "pathologie dans la prestation des documents $»$.

II serait peut-être possible de diminuer les échecs des lecteurs à trouver les volumes connus qu'ils cherchent en leur suggérant des titres équivalents; mais, comme le fait remarquer Buckland ${ }^{9}$ lui-même, la pertinence d'une alternative de ce genre est difficile à évaluer présentement, étant donné notre ignorance du processus de «substitution » des titres dans les bibliothèques d'enseignement et de la proportion des lecteurs qui prennent la peine de signaler aux bibliothécaires de référence les échecs rencontrés dans leur recherche de volumes précis.

Buckland conclut sa recherche en affirmant que la règle fondamentale de la gestion de la collection d'une bibliothèque (library stock control ) consiste à relier la période de prêt et la politique de duplication au niveau de popularité de chaque titre. La possibilité de modifier la durée de la période de prêt constitue un moyen efficace d'exercer une influence sur la disponibilité des volumes de la collection et d'accroître, par conséquent, le «niveau de satisfaction ".

\section{«Exposition aux documents» de Hamburg}

Hamburg 10 et d'autres chercheurs ont proposé comme mesure des résultats d'une bibliothèque un système qu'ils appellent l'« exposition aux documents " (document exposure). Hamburg soutient que l'objectif fondamental des bibliothèques publiques et universitaires est de favoriser au maximum le contact des usagers avec l'information enregistrée et que, par conséquent, la mesure de performance la plus appropriée pour évaluer l'efficacité de ces bibliothèques est le calcul du nombre de documents consultés par la clientèle, c'est-àdire l'«exposition aux documents ».

Même si la mesure de performance proposée par Hamburg ne tient pas compte de la

9. Ibid.

10. Morris Hamburg et al., Library-Planning and Decision-Making Systems, Cambridge, Mass., M.I.T. Press, 1974, $274 p$ proportion des requêtes documentaires satisfaites, elle vaut la peine d'être soulignée, car elle témoigne de l'absence de consensus dans la profession sur les objectifs majeurs d'une bibliothèque et, par conséquent, sur les mesures de performance à utiliser pour évaluer leur degré de réalisation.

La méthodologie retenue par Hamburg pour mesurer l'"exposition aux documents " consiste à compter chaque prêt (et chaque renouvellement), chaque document consulté à l'intérieur de la bibliothèque, chaque prêt entre bibliothèques et chaque consultation indirecte, c'est-à-dire chaque document consulté par un bibliothécaire de référence pour trouver l'information demandée par un usager, comme une unité d'exposition. Toutes les unités d'exposition sont ensuite additionnées pour former une mesure globale.

Dans un article ${ }^{11}$ paru antérieurement, Hamburg avait déjà exposé l'essentiel de son projet; il consiste en trois mesures de performance basées sur l'«exposition aux documents ». Ces trois mesures sont: le calcul des expositions, le nombre d'expositions-jours et le temps d'exposition. Nous n'étudierons pas plus en détail le modèle analytique élaboré par Hamburg. En effet, en se limitant à mesurer quantitativement le volume d'utilisation d'une bibliothèque, il ne peut être utile que dans une étude de macroévaluation. Un tel modèle ne constitue pas un outil diagnostique permettant d'identifier les facteurs qui affectent le volume d'utilisation d'une bibliothèque et d'améliorer, à la lumière des résultats de l'analyse, son efficacité dans l'avenir.

\section{«Etude statistique de la circulation des volumes" de Morse}

Même s'il ne vise pas, à proprement parler, à étudier la proportion des requêtes de documents identifiés qu'une bibliothèque peut satisfaire, nous ne pouvons passer sous silence le volume de Philip Morse ${ }^{12}$, nettement supérieur

11. Morris Hamburg et al., "Library objectives and performance measures and their use in decisionmaking ". Library Quarterly, vol. 42. no. 1 (January 1972). 107-128.

12. Philip Morse, Library Effectiveness. A Systems Approach, Cambridge, Mass., M.I.T. Press, 1968, $207 \mathrm{p}$. 
aux travaux de Hamburg. L'étude de Morse, qui a obtenu le "Lanchester Prize» de la "Operations Research Society of America » en 1968, est déjà considérée comme un classique de la bibliothéconomie scientifique.

Dans cette étude, Morse applique les techniques de la recherche opérationnelle au domaine de la circulation des volumes. II se sert de modèles probabilistes pour prédire l'utilisation moyenne future des volumes d'une classe donnée. Ses modèles mathématiques ont comme fondement conceptuel la théorie des probabilités et s'avèrent fort sophistiqués. D'ailleurs, il n'est guère possible, sans de solides connaissances en techniques statistiques, de suivre les développements théoriques et empiriques du volume de Morse.

En 1972, Morse a publié un article ${ }^{13}$ un peu plus accessible dans lequel il modifie et améliore ses modèles probabilistes qui avaient été critiqués par Jain ${ }^{14}$. Nous nous baserons sur ce texte pour exposer la problématique de Morse.

II utilise une approche analytique dans le but de prédire la circulation moyenne future des volumes d'une discipline. A partir d'un échantillonnage probabiliste, il recueille des données sur l'historique de la circulation de monographies appartenant à la classe en cours d'étude, en examinant les cartes de prêt des volumes placés sur les rayons ainsi que les cartes des volumes actuellement empruntés. L'analyse statistique des données recueillies permet de calculer la circulation moyenne des volumes de la discipline ainsi que la proportion des volumes qui ont été empruntés une fois ou plus (volumes actifs) durant l'année. La proportion des nouveaux volumes qui ont été actifs durant l'année ainsi que leur circulation moyenne constituent des mesures d'efficacité de la politique de choix des monographies.

13. Philip Morse, "Measures of library effectiveness", Library Quarterly, vol. 42, no. 1 (January 1972), 1530.

14. Sur cette question, voir Ching-Chih Chen, Applications of Operations Research Models to Libraries. A Case Study of the Use of Monographs in the Francis A. Countway Library of Medicine, Harvard University, Cambridge, Mass., M.I.T. Press, 1976, p. 7.
Les données sur l'historique de la circulation des volumes d'une discipline permettent de prédire leur circulation dans l'avenir, à condition, bien sûr, d'être intégrées dans un modèle mathématique adéquat. En effet, plusieurs études ont démontré que la circulation des monographies est reliée à leur circulation durant les années précédentes. Le modèle mathématique qui permet de prédire la circulation future moyenne des volumes d'une discipline en fonction de leur circulation antérieure est un modèle stochastique de type markovien (chaîne de Markov) intégrant les variables suivantes:

$R(t+1)=a+B R(t)$

$\mathrm{a}=$ paramètre indiquant la circulation moyenne des plus vieux volumes de la classe étudiée;

$B=$ paramètre mesurant la perte de popularité des volumes de cette classe d'année en année;

$R(t)=$ circulation moyenne des volumes de la discipline durant l'année écoulée;

$R(t+1)=$ circulation moyenne prévisible durant la prochaine année.

Les valeurs des paramètres a et $B$, qui indiquent la diminution de la circulation des volumes en fonction de leur "âge", changent très peu d'année en année et n'ont donc pas besoin d'être réévaluées plus d'une fois à tous les quatre ou cinq ans. Ces paramètres s'avèrent d'une grande utilité puisqu'ils sont intégrés dans une chaîne de Markov. Ils fournissent les informations requises pour prendre des décisions rationnelles au moment d'adopter des politiques concernant les services offerts aux usagers; ils permettent, notamment, de déterminer d'une manière rationnelle et éclairée quand il convient de se procurer un exemplaire supplémentaire d'un titre et de procéder à l'élagage des volumes inactifs.

Les modèles probabilistes construits par Morse et expérimentés par lui à la Bibliothèque des sciences du Massachusetts Institute of Technology (M.I.T.) ont également été vérifiés par Chen ${ }^{15}$ à la Bibliothèque de médecine de l'Université de Harvard. Chen considère l'analyse statistique de la circulation des monogra-

15. Ibid., 207. 
phies comme une entreprise très importante, parce que l'utilisation d'un volume est, selon lui, une mesure objective de l'efficacité d'une bibliothèque, indiquant qu'un document a satisfait un besoin 16 .

Pour tester la capacité des modèles de Markov à prédire la circulation moyenne des volumes d'une discipline donnée, Chen a utilisé une procédure différente de celle employée par Morse pour recueillir des données sur la circulation des monographies. II s'est servi uniquement des cartes de livres placées au comptoir de prêt lorsqu'ils sont empruntés par les usagers. II est évident que les données recueillies de cette façon sont biaisées en faveur des volumes les plus actifs de la classe étudiée; elles ne sont pas représentatives des volumes qui sont peu ou jamais empruntés. Pour rectifier cette situation, Chen a été obligé d'employer des facteurs de correction qui compliquent davantage l'analyse statistique de la circulation des volumes. Dans un article rédigé en collaboration avec Morse, Chen ${ }^{17}$ avoue qu'il est préférable de se servir des cartes de prêt des volumes placés sur les rayons pour éviter d'entreprendre une étude avec un échantillonnage biaisé.

Grâce à l'emploi de facteurs de correction, Chen est parvenu à démontrer, lui aussi, la capacité des chaînes de Markov à prédire la circulation moyenne future des volumes d'une classe. C'est donc dire que les modèles théoriques construits par Morse ont été vérifiés avec succès dans deux bibliothèques importantes. Dans les deux expériences, la circulation moyenne théorique prédite par le modèle mathématique a correspondu sensiblement à la circulation empirique (réelle).

II est important de souligner que les modèles mathématiques élaborés par Morse s'appliquent dans n'importe quel genre de bibliothèque. Les valeurs mathématiques des paramètres diffèrent d'une discipline à l'autre et d'une bibliothèque à l'autre. De plus, les valeurs des paramètres d'une même classe peuvent varier considérablement quand elles sont calculées

16. Ibid., 67.

17. Philip Morse and Ching-Chih Chen, "Using circulation desk data to obtain unbiased estimates of book use », Library Quarterly, vol. 45, no. 2 (April 1975), 193. dans des bibliothèques différentes. C'est pourquoi les paramètres requis pour appliquer les modèles probabilistes de Morse doivent être calculés pour chaque classe d'une bibliothèque particulière.

\section{«Frustration de l'usager» de Saracevic}

Le modèle d'évaluation élaboré par Saracevic ${ }^{18}$ et ses collaborateurs tente de mesurer la performance d'une bibliothèque au moyen d'une analyse mathématique et diagnostique des requêtes de documents identifiés, satisfaites et insatisfaites (produisant la frustration de l'usager ); il s'inspire de l'étude de Buckland analysée plus haut. Alors que Buckland étudiait principalement le degré de corrélation existant entre la politique de circulation et la disponibilité des volumes, Saracevic considère la politique de prêt comme étant seulement un des facteurs qui causent la satisfaction ou la frustration de l'usager. C'est pourquoi son modèle analytique tient compte de quatre facteurs majeurs qui affectent la performance d'une bibliothèque mesurée par le «niveau de satisfaction ».

Saracevic identifie les variables susceptibles d'engendrer de la frustration chez les usagers; ce sont le service des acquisitions, la politique de prêt, le fonctionnement de la bibliothèque et le comportement même de l'usager. Ces quatre facteurs sont objectivement mesurables et constituent des paramètres significatifs de la qualité du service offert par une bibliothèque.

Saracevic a appliqué son modèle analytique à la Bibliothèque Sears de la Case Western Reserve University en 1972 et en 1974, dans le but de mesurer les principaux facteurs qui causent la satisfaction ou la frustration de la clientèle et d'évaluer la capacité de cette bibliothèque de satisfaire les requêtes de documents identifiés, par l'intermédiaire de l'indicateur «niveau de satisfaction». Les résultats de l'enquête effectuée en 1972 furent les suivants:

a) mesure de performance des acquisitions: $88 \%$;

18. Tefko Saracevic, W.M. Shaw jr and B.P. Kantor, "Causes and dynamics of user frustration in an academic library", College and Research Libraries, vol. 38, no. 1 (January 1977), 7 - 18. 
b) mesure de performance de la politique du prêt: $77 \%$;

c) mesure de performance du fonctionnement de la bibliothèque: $89 \%$;

d) mesure de performance des usagers : $80 \%$;

e) "niveau de satisfaction»: $48 \%$.

La faible performance de la Bibliothèque Sears concorde avec les études antérieures qui avaient démontré qu'un "niveau de satisfaction » variant entre $40 \%$ et $60 \%$ est très fréquent dans la plupart des bibliothèques de collèges et d'universités. II est très important de souligner que, dans le modèle analytique de Saracevic, la bibliothèque a la possibilité de modifier les quatre paramètres affectant d'une manière significative le "niveau de satisfaction » et peut ainsi améliorer sa performance. L'efficacité du service des acquisitions peut être accrue en élaborant, entre autres, une politique plus rationnelle de choix des documents et en choisissant les fournisseurs offrant un service plus rapide. L'efficacité du service de prêt peut être augmentée par une réduction de la durée de la période de prêt, ce qui assure une plus grande disponibilité des volumes. Le fonctionnement interne de la bibliothèque peut être amélioré en remettant quotidiennement les volumes sur les rayons, en faisant des lectures de rayons plus fréquentes, en faisant des inventaires réguliers et en implantant un système anti-vol, puisqu'il a été prouvé que le vol des volumes constitue une des causes majeures de la frustration des usagers.

La mesure de performance des usagers à la Bibliothèque Sears en 1972 a été de 80\%. Cela signifie que de tous les volumes qui ont fait l'objet de requêtes par la clientèle et qui étaient disponibles, c'est-à-dire qui avaient été acquis, n'avaient pas été empruntés et étaient bien placés sur les rayons, 20\% n'ont pu être trouvés à cause d'erreurs réalisées par les usagers eux-mêmes. Même si la déficience est le fait de l'usager et non de la bibliothèque, cette dernière peut améliorer l'efficacité de l'usager et, conséquemment la sienne, en mettant sur pied un programme adéquat d'initiation des usagers à l'utilisation de la bibliothèque, en plaçant au catalogue et sur les rayons des signaux de localisation qui aident l'usager à découvrir la notice ou le volume recherché.

\section{«Temps global d'exposition par usager potentiel » de Kantor}

Le modèle analytique construit par Kantor ${ }^{19}$ intègre deux mesures de performance étudiées plus haut, soit le "temps d'exposition" de Hamburg et le "niveau de satisfaction » de Buckland. Kantor fusionne ces deux mesures en une seule qu'il désigne sous le nom de "temps global d'exposition par usager potentiel » (total contact time per potential user). Cette dernière mesure est obtenue au moyen d'un modèle fort complexe comprenant huit paramètres significatifs, objectivement mesurables et plus ou moins manipulables.

\section{Modèle à utiliser dans une auto-évaluation}

Les modèles évaluant la capacité d'une bibliothèque de satisfaire les requêtes de documents identifiés sont basés sur deux types d'échantillonnage: a) un échantillon de titres provenant d'une bibliographie quelconque; b) un échantillon de titres choisis parmi les demandes documentaires formulées par les usagers de la bibliothèque évaluée. Le test de «livraison de documents » de Orr ainsi que l'instrument d'évaluation de De Prospo 20, lequel se base sur un échantillonnage de 500 notices bibliographiques récentes signalées dans American Book Publishing Record et évalue quelle proportion de ces titres est disponible sur les rayons d'un certain nombre de bibliothèques publiques américaines, utilisent le premier type d'échantillonnage. Or, celui-ci est beaucoup moins valable que le second, puisqu'il ne tient pas compte de la clientèle spécifique de chaque bibliothèque. En effet, il est impossible de démontrer qu'un échantillon choisi au hasard de volumes récemment signalés dans Publishers' Weekly par exemple constitue un instrument d'évaluation adéquat et représentatif des besoins documentaires de la clientèle d'une bibliothèque particulière.

Un échantillon élaboré à partir des deman-

19. B.P. Kantor, "The library as an information utility in the university context: Evolution and measurement of service", Journal of the American Society for Information Science, vol. 27, no. 2 (March-April 1976), 100-112.

20. Ernest R. De Prospo, E. Altman and Kenneth E. Beasley, Performance Measures for Public Libraries, Chicago, American Library Association, 1973,.77 p. 
des réelles des usagers est beaucoup plus valable que celui basé sur des références bibliographiqes supposément représentatives des besoins réels et actuels de la clientèle de toute bibliothèque. De tous les modèles présentement disponibles qui utilisent les requêtes documentaires réelles des usagers, nous recommandons l'emploi du modèle analytique construit par Saracevic. Ce modèle permet une analyse diagnostique de la capacité d'une bibliothèque particulière de satisfaire les besoins en information exprimés par les usagers et il est suffisamment simple pour être appliqué, non par des spécialistes de la recherche opérationnelle, mais par le personnel même de la bibliothèque qui veut procéder à son autoévaluation.

II est évident que le modèle de Saracevic, même s'il procède à une microévaluation d'un des services les plus importants de la bibliothèque, ne constitue pas un instrument d'évaluation parfait. II ne tient compte que des besoins exprimés et ignore les besoins non exprimés (demandes latentes). Les besoins documentaires non exprimés sont d'ailleurs très difficiles à déterminer parce qu'ils sont le résultat de perceptions de la clientèle potentielle. Les non-usagers ne prennent pas la peine d'exprimer des requêtes documentaires parce qu'ils ne croient pas la bibliothèque capable de satisfaire leurs besoins.

\section{Evaluation de «l'efficacité de repérage"}

Evaluer «l'efficacité de repérage » ( to measure retrieval effectiveness) d'une bibliothèque consiste à déterminer si les produits documentaires fournis aux usagers qui expriment des requêtes par sujet satisfont effectivement leurs exigences. Les produits ou les résultats d'une recherche documentaire, peu importe qu'elle soit effectuée dans un catalogue sur fiches, dans un index de périodiques ou dans une banque de données bibliographiques au moyen d'un terminal, peuvent être des références, des résumés analytiques ou encore les documents eux-mêmes. Une microévaluation, une analyse diagnostique de "l'efficacité de repérage " a pour but d'évaluer la qualité des produits communiqués aux usagers et d'identifier les facteurs qui affectent les résul- tats de ce service, l'un des principaux offerts par les bibliothèques.

Les études d'évaluation de l'efficacité des recherches documentaires par sujet effectuées dans les catalogues sur fiches des bibliothèques ont malheureusement été dénuées de toute rigueur scientifique jusqu'à maintenant. Comme le souligne Lancaster ${ }^{21}$, ces études ont été tout à fait superficielles puisqu'elles ne visaient pas à déterminer la proportion des documents pertinents repérés, mais se limitaient plutôt à vérifier si l'usager avait découvert une vedette-matière appropriée à son sujet ou encore s'il avait choisi un volume sur les rayons à la suite de sa démarche.

Face à l'inexistence en bibliothéconomie de recherches rigoureuses portant sur l'évaluation de l'efficacité des recherches documentaires, les bibliothécaires ont intérêt à analyser les nombreuses études sur le sujet entreprises dans les centres de documentation (information retrieval systems). En sciences de l'information 22 stricto sensu, l'évaluation de l'efficacité des centres de documentation constitue un des secteurs de recherche les plus dynamiques et se base justement sur des mesures de repérage ( retrieval measures). Les deux mesures de performance les plus fréquemment employées par les spécialistes des sciences de l'information pour évaluer "l'efficacité de repérage » des systèmes documentaires sont le rappel (recall) et la précision (precision). Or ces deux mesures 23 constituent des critères très valables pour évaluer les résultats des recherches documentaires faites dans les catalogues sur fiches des bibliothèques.

\section{Mesures de rappel et de précision}

Le rappel et la précision sont deux mesures quantitatives qui permettent d'évaluer la qualité, le résultat d'une recherche documentaire effectuée dans un index de périodiques, dans

21. F.W. Lancaster, The Measurement and Evaluation..., p. 162.

22. Rowena Weiss Swanson, "Performing evaluation studies in information science", Journal of the American Society for Information Science, vol. 26, no. 3 (May-June 1975), 140.

23. F.W. Lancaster, The Measurement and Evaluation..., p. 148. 
une banque de données bibliographiques ou encore dans un catalogue sur fiches. La mesure de rappel d'une recherche manuelle ou automatisée est la proportion des documents pertinents contenus dans le système qui ont été repérés. La précision est la proportion des documents repérés qui sont pertinents à la requête. Ces deux mesures sont exprimées sous la forme d'un pourcentage calculé comme suit:

$$
\begin{aligned}
& \text { Rappel }= \begin{array}{l}
\text { le nombre de documents } \\
\text { pertinents repérés }
\end{array} \\
& \begin{array}{l}
\text { le nombre total de documents } \\
\text { pertinents contenus dans le } \\
\text { système }
\end{array} \\
& \text { Précision }= \\
& \begin{array}{l}
\text { le nombre de documents } \\
\text { le nombre total de docu- } \\
\text { ments repérés }
\end{array}
\end{aligned}
$$

Le rappel et la précision sont deux mesures étroitement liées; elles perdent leur signification quand elles sont utilisées isolément. Lorsque ces deux statistiques de performance sont considérées ensemble 24 , elles constituent des indicateurs valables de la capacité de filtrage d'un système documentaire, de son efficacité à ne repérer que des documents pertinents aux requêtes exprimées par sujet. Pour déterminer l'indicateur de performance global de rappel et de précision d'un système documentaire, il suffit de calculer le pourcentage de rappel et de précision de chacune des recherches documentaires effectuées et d'établir, par la suite, la moyenne de tous les résultats individuels obtenus.

Le rappel est une mesure efficace du succès d'une recherche documentaire, que l'usager effectue lui-même la recherche ou qu'il la délègue à un documentaliste. Cependant, il faut préciser que le rappel constitue un indicateur de très grande importance quand l'usager a besoin d'une bibliographie exhaustive sur un sujet. Un niveau maximum de rappel prend toute sa signification uniquement dans le cas de recherches documentaires menées dans des centres de documentation

24. F.W. Lancaster, "Evaluation and testing of information retrieval systems", in Allen Kent and Harold Lancour (eds.), Encyclopedia of Library and Information Science, vol. 8, New York, Marcel Dekker, 1972 , p. 236. au profit de chercheurs intéressés à consulter le plus grand nombre possible de documents parus sur un sujet.

La majorité des recherches sont réalisées par ou pour des usagers intéressés à obtenir, non pas tous les documents pertinents contenus dans un système, mais un certain nombre de documents parmi les plus récents parus sur le sujet. Cette situation se présente d'ailleurs dans les bibliothèques où les usagers n'exigent habituellement pas un niveau très élevé de rappel, mais un niveau minimum pouvant aller de un à cinq documents pertinents.

Pour les lecteurs qui n'ont besoin que de quelques documents sur un sujet, la précision constitue sans doute la plus importante mesure de l'efficacité d'une recherche documentaire. II faut néanmoins ajouter que cette mesure de performance est beaucoup plus significative dans le cas d'une recherche déléguée à un bibliothécaire ou à un documentaliste que lorsque l'usager l'effectue luimême. Lorsqu'il s'agit d'une recherche déléguée, l'usager usant de la mesure de précision pour évaluer l'efficacité de la recherche utilise ainsi un critère fort valable. Cependant, une condition est essentielle pour assurer la validité de l'évaluation: les résultats de la recherche communiqués au lecteur afin que celui-ci en détermine le niveau de précision ne doivent pas avoir été filtrés par le documentaliste. En d'autres mots, la précision doit être calculée par l'usager sur la base des résultats bruts de la recherche et non à partir des résultats filtrés par un professionnel du centre de documentation.

Dans le cas d'une recherche documentaire effectuée par l'usager lui-même, Lancaster 25 souligne que la précision ne constitue pas une mesure très significative de l'efficacité de la recherche. II propose de remplacer la mesure de précision par le calcul du temps requis par l'usager pour effectuer sa recherche. Le temps pris par le lecteur est ensuite divisé par le nombre total de références jugées pertinentes afin de déterminer le coût unitaire (en temps) de chaque référence pertinente repérée. La durée de la recherche fournit une mesure plus

25. F.W. Lancaster, The Measurement and Evaluation..., p. 145. 
adéquate de l'effort demandé à l'usager et tient d'ailleurs compte de la mesure de précision: en effet, plus la précision (proportion des références pertinentes sur le nombre total de références examinées) est grande, plus la durée de la recherche est courte.

Les mesures de rappel et de précision ont comme fondement la pertinence des documents repérés sur un sujet particulier. Or, la pertinence d'un document est une réalité fort complexe qui fait encore l'objet de vives controverses entre les spécialistes des sciences de l'information. Dans un article récent, Saracevic 26 fait le point d'une façon remarquable sur ce concept difficile à appréhender. La pertinence d'un document est une réalité subjective, car l'usager attribue cette qualité à un volume sans démontrer d'une manière objective le bien-fondé de son affirmation. C'est donc dire que chaque lecteur peut interpréter différemment la pertinence ou la non-pertinence d'un document en relation avec un besoin précis en information.

Comme la pertinence est un phénomène subjectif, il faut en déduire que seule la personne qui exprime la requête documentaire est en mesure de juger de la pertinence des documents qui sont repérés. Même si la pertinence n'est pas une réalité susceptible de se prêter à une analyse objective, elle constitue néanmoins un concept fondamental de l'évaluation de "l'efficacité de repérage"; en effet, il est évident que l'objectif majeur des systèmes documentaires (bibliothèques ou centres de documentation ) est de communiquer à la clientèle l'information pertinente dont elle a besoin.

\section{«Projet Cranfield» de Cleverdon}

Après avoir élaboré de façon sommaire le cadre conceptuel d'une étude d'évaluation de "l'efficacité de repérage", nous examinerons les réalisations dans ce domaine. Nous nous limiterons à l'analyse de la première étude majeure portant sur l'évaluation de l'efficacité des systèmes documentaires, le «Projet Cran-

26. Tefko Saracevic, "Relevance a review of the literature and a framework for thinking on the notion in information science", in Melvin J. Voigt and Michael $\mathrm{H}$. Harris (eds.), Advances in Librarianship, vol. 6, New York, Academic Press, 1976, p. 79-138. field » réalisé par Cleverdon 27 et une équipe de spécialistes. Comme le fait remarquer Saracevic ${ }^{28}$, le "Projet Cranfield » a retenu et retient toujours l'attention des experts de l'évaluation en sciences de l'information. II est toujours d'actualité, puisque toutes les recherches empiriques portant sur l'évaluation de l'efficacité des «information retrieval systems " réalisées jusqu'à ce jour sont basées sur le cadre théorique et méthodologique élaboré par Cleverdon. De plus, les débats controversés qui ont débuté dès la parution des résultats de la recherche de Cleverdon se poursuivent toujours aujourd'hui et portent essentiellement sur la méthodologie utilisée dans les tests d'évaluation.

La recherche de Cleverdon avait pour objectif de déterminer le degré d'efficacité des divers langages documentaires employés pour indexer les documents contenus dans les systèmes d'information enregistrée. Pour réaliser cet objectif, Cleverdon s'est servi d'une collection de 1,200 documents en aérodynamique. Un total de 280 questions formulées par des experts qui avaient déjà publié des écrits sur ce sujet ont été adressées au centre de documentation expérimental soumis à l'évaluation. II avait été convenu au départ que la pertinence des documents repérés en réponse aux requêtes serait jugée par les experts euxmêmes.

Dans le but de mesurer le rappel et la précision des résultats obtenus pour chacune des requêtes, les auteurs de la recherche ont décidé d'examiner chaque document afin de déterminer ceux qui étaient pertinents à chacune des 280 questions. En connaissant à l'avance les références pertinentes à une requête, il devenait facile de vérifier lesquelles, parmi les méthodes d'indexation évaluées, avaient ou n'avaient pas repéré les documents pertinents contenus dans la collection expérimentale.

Comme l'examen des rapports de pertinence entre les 1,200 documents et les 280

27. Cyril Cleverdon, J. Mills and Michael Keen, ASLIB Cranfield Research Project. Factors Determining the Performance of Indexing Systems, vol. 1. Test Design, Cranfield, Berfordshire, England, College of Aeronautic, 1966.

28. Tefko Saracevic, "Relevance: a review...", 98. 
questions représentait une tâche trop lourde pour être exécutée par les experts euxmêmes, Cleverdon engagea cinq étudiants post-gradués en aérodynamique pour filtrer les documents et communiquer aux experts seulement ceux qui leur paraissaient pertinents aux requêtes. Les étudiants ont retenu 592 documents et les ont soumis aux experts qui avaient formulé les 280 questions. Or, l'étude d'un échantillonnage de 129 documents pertinents à une des questions posées a permis de découvrir que les étudiants n'en avaient effectivement retenu que dix, ce qui représente un faible pourcentage de $8 \%$. Si cet échantillon est représentatif de l'efficacité du filtrage effectué par les étudiants, il faut en déduire que ces derniers ont raté 7,600 références qui avaient pourtant été jugées pertinentes aux 280 requêtes. Les raisons pouvant expliquer la piètre performance des étudiants n'ont jamais été explicitées.

En engageant des étudiants pour filtrer les documents potentiellement pertinents, Cleverdon violait son propre critère de validité méthodologique qui spécifiait que la pertinence devait être jugée uniquement par l'expert qui avait formulé la question. Comme nous l'avons souligné plus haut, la pertinence est un phénomène subjectif et doit, par conséquent, être déterminée uniquement par l'usager qui fait la demande documentaire. Dans une analyse critique vigoureuse du "Projet Cranfield", Don R. Swanson 29 conclut que le filtrage des documents possiblement pertinents par des étudiants constitue une déficience méthodologique grave qu'il désigne sous le nom «d'erreur de la recherche déléguée » (fallacy of delegation ).

La recherche de Cleverdon comporte une lacune méthodologique suffisamment sérieuse pour jeter le doute sur la validité des résultats obtenus. Les bibliothécaires et les documentalistes qui utilisent, sans aucune analyse critique, les résultats du "Projet Cranfield» pour affirmer la supériorité d'une procédure d'indexation (l'indexation à vocabulaire libre par exemple) sur une autre, se basent sur une recherche dont la rigueur scientifique est fortement contestée. Nous sommes donc obli-

29. Don R. Swanson, "Information retrieval as a trialand-error process ", Library Quarterly, vol. 47, no. 2 (April 1977), 134. gés de constater l'absence de connaissances rigoureuses dans le domaine de l'évaluation de "l'efficacité de repérage», car il n'existe présentement aucune étude empirique sur le sujet qui soit exempte de déficiences méthodologiques.

\section{Analyse diagnostique des déficiences dans le rappel et la précision}

Les résultats des mesures de rappel et de précision dans les bibliothèques et dans les centres de documentation dépendent d'un nombre considérable de facteurs (variables). L'identification et le contrôle des variables majeures qui affectent "l'efficacité de repérage » des systèmes documentaires constituent des prérequis indispensables à toute tentative d'amélioration de leur performance. Les principaux facteurs agissant sur "l'efficacité de repérage» sont, entre autres, la formulation de la requête, le langage documentaire, la qualité et la cohérence de l'indexation, le degré de spécificité des descripteurs ou des vedettes-matière, la stratégie de recherche. Les futurs projets de microévaluation, d'analyse diagnostique de l'efficacité des recherches documentaires menées dans les bibliothèques du Québec devront identifier et mesurer les causes des déficiences de rappel (recall failures) ainsi que les causes des déficiences de précision (precision failures ), pour aboutir à des améliorations significatives de la qualité de ces recherches.

\section{Conclusion}

L'évaluation objective de la capacité d'une bibliothèque de satisfaire les multiples besoins de sa clientèle requiert l'emploi d'indicateurs de résultats. II faut préciser que nous avons fait référence, tout au long de cet article, à des indicateurs internes et non à des indicateurs externes. En effet, le fonctionnement d'une bibliothèque implique la réalisation de deux types de résultats: les résultats internes et les résultats externes. Les résultats internes se rapportent aux produits et aux services documentaires offerts à la clientèle d'une bibliothèque particulière; à ces résultats de premier niveau sont reliés des indicateurs d'efficacité qui révèlent dans quelle mesure les produits et les services réalisés correspondent 
aux objectifs documentaires poursuivis et satisfont les besoins en information des usagers.

Les résultats externes concernent la somme des avantages que les usagers retirent de l'utilisation d'une bibliothèque. A ces résultats de deuxième niveau correspondent des indicateurs de "valeur " qui révèlent les effets de la bibliothèque sur son environnement. Les indicateurs de résultats de deuxième niveau visent à mesurer la contribution de la bibliothèque (système ouvert) à la réalisation des objectifs des systèmes faisant partie de son environnement. Présentement, les indicateurs existant en bibliothéconomie sont tous des indicateurs d'efficacité. La construction d'indicateurs de résultats externes qui rendront possible la mesure objective des bénéfices pédagogiques, culturels, technologiques, économiques que retirent les utilisateurs d'une bibliothèque s'avère une tâche extrêmement complexe.

Après ce rapide survol des principales études de performance réalisées en bibliothéconomie, plusieurs praticiens ont peut-être l'impression qu'un projet d'évaluation est une entreprise essentiellement théorique et académique qui n'a rien à voir avec le fonctionnement quotidien d'une bibliothèque. Or, il n'en est rien. Une étude objective de l'efficacité d'une bibliothèque est réalisée par des praticiens qui se préoccupent essentiellement d'améliorer la qualité des services offerts à la clientèle. Un projet d'évaluation est avant tout une recherche orientée vers l'action, une entreprise de lecture rigoureuse et systématique de la vie d'une bibliothèque qui ne peut conduire qu'à une évolution de sa pratique quotidienne.

Une institution qui ne subit pas d'évaluations systématiques est aveugle. II n'y a rien de plus nuisible à l'image d'une profession que des praticiens qui n'ont que des opinions sur l'efficacité des services à leur clientèle. La population n'est-elle pas justifiée de demander aux bibliothécaires et aux documentalistes de tester par des méthodes rigoureuses et scientifiques l'efficacité de leur pratique quotidienne?

L'amélioration de l'efficacité d'une bibliothèque exige un diagnostic sérieux de ses déficiences majeures. Or, un véritable diagnostic ne repose pas sur des opinions ou sur des impressions subjectives, mais sur une analyse objective approfondie d'une situation réelle en vue de proposer des solutions rationnelles à des problèmes concrets.

La mesure objective des résultats de la bibliothèque bénéficiera non seulement à la clientèle, mais aux bibliothécaires eux-mêmes. Depuis quelques années, plusieurs bibliothèques ont subi des coupures budgétaires considérables. Ces pertes financières ont-elles diminué l'efficacité des bibliothèques? Nous sommes impuissants à le démontrer parce que nous ne quantifions pas l'efficacité de nos services; nous sommes, par conséquent, incapables de prouver, chiffres à l'appui, la baisse de performance et la diminution sensible dans la qualité des services offerts aux usagers qu'ont provoquées ces coupures budgétaires.

La quantification ou la mesure objective de l'efficacité des divers services offerts par une bibliothèque à sa clientèle ne peut être que fragmentaire. En effet, la seule approche raisonnable de l'évaluation d'une bibliothèque consiste à mesurer chacun de ses services d'une manière différente et séparée ${ }^{30}$. II est évident, par exemple, que le service de référence, le service des périodiques et les réponses fournies aux usagers qui expriment des requêtes documentaires précises ne peuvent être évalués de la même façon: la méthodologie et les indicateurs de performance vont varier d'un service à l'autre.

La mesure de l'efficacité de tous lès services offerts par la bibliothèque à ses usagers va exiger la construction et la validation de nombreux modèles analytiques. Ces modèles devront s'appliquer à toutes les catégories de bibliothèques et permettre d'obtenir un grand nombre d'indicateurs de performance documentaire. Dès que la validité d'un modèle aura été établie, la détermination des valeurs à attribuer aux divers paramètres pour une bibliothèque particulière s'avérera une tâche relativement simple en comparaison de la construction d'un nouveau modèle.

Le puissant langage des modèles utilisés dans l'approche systémique ou la recherche

30. F.W. Lancaster, The Measurement and Evaluation..., p. 372. 
opérationnelle permet, par un simple calcul, de quantifier et de mesurer l'efficacité d'un système documentaire. Comme la mesure est le fondement même de toute science, l'emploi de méthodes quantitatives permettra à la fois de quantifier la performance des bibliothèques et de modifier l'image de la bibliothéconomie, encore perçue dans de nombreux milieux comme un recueil d'opinions et de recettes. Si la bibliothéconomie scolaire et académique peut réussir à contrer l'impérialisme de la pédagogie, tous les types de bibliothèques pourront s'orienter vers une conception et une pratique scientifique des fonctions documentaires. La bibliothéconomie quantitative et statistique permettra d'éviter que l'Association pour l'avancement des sciences et des techniques de la documentation n'ait de scientifique que le nom.

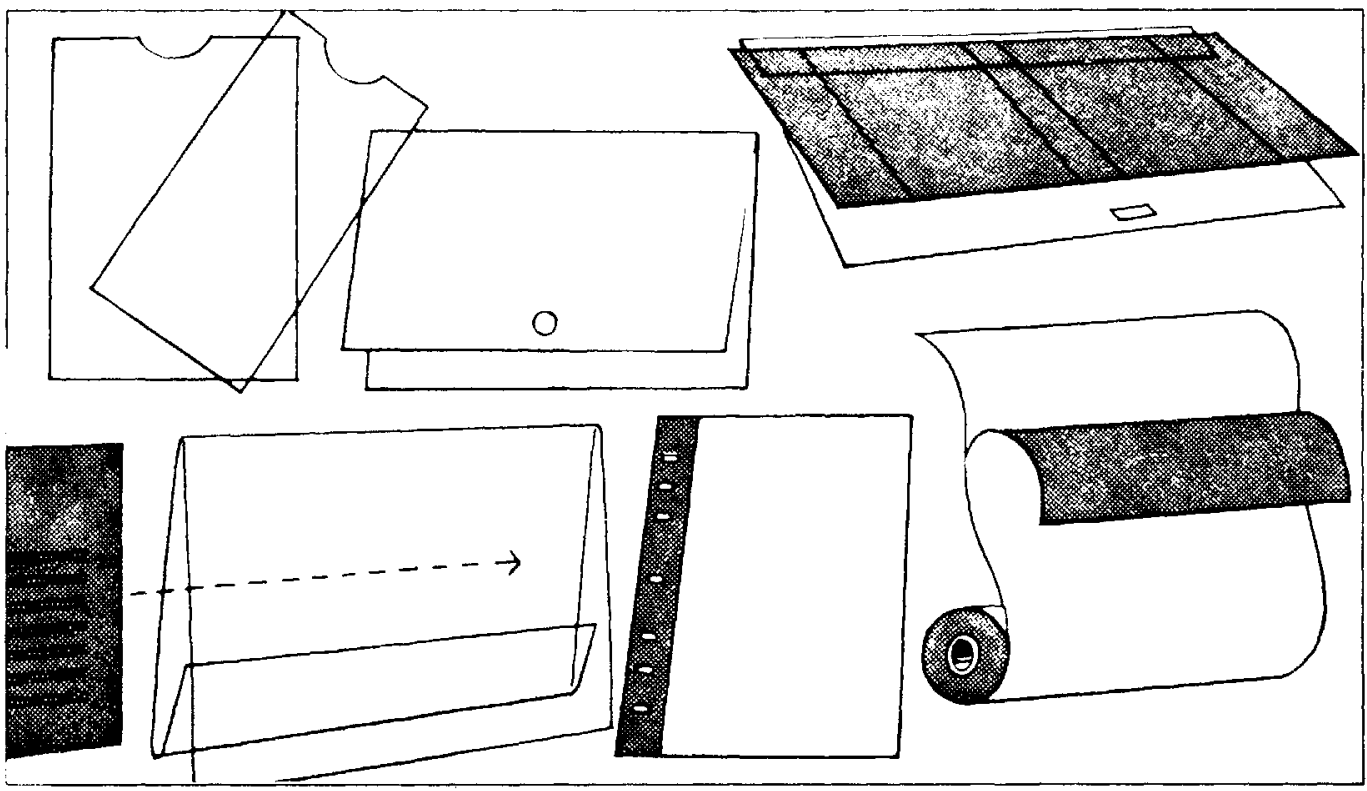

PROTECTION PLASTIQUE - PROTEGGE-DOCUMENTS - MATERIEL DE REPARATIONS

R.M.

DEMANDEZ NOTRE CATALOGUE 78

LEDUC

\& CIE 2101, RUE BENNETT - MONTRÉAL H1V 2T4 - TEL.: 255-1221 